\title{
artigo
}

Martins, J.F.M.S.; Santos, J.R.F.M.; Rocha, L.A.; Franco, M.S.; Amorim, L.S.; Lima, L.H.O.;

Intervenção educativa para idosos hipertensos: um relato de experiência

\section{Intervenção educativa para idosos hipertensos: um relato de experiência}

\author{
Educational intervention for hypertensive elderly: an experience report \\ Intervención educativa para ancianos hipertensos: informe de uma experiencia
}

\begin{abstract}
RESUMO
OBJETIVO: Descrever a experiência de intervenção educativa em saúde para controle da Hipertensão Arterial (HAS) em idosos. MÉTODOS: Estudo descritivo, tipo relato de experiência, de ações de intervenção educativas desenvolvidas com um grupo de idosos hipertensos, entre março a julho de 2019 em uma UBS no município de Paulistana - PI. RESULTADOS: Foram realizados 8 encontros: três palestras sobre hábitos alimentares saudáveis no controle dos níveis pressóricos; três atendimentos nutricionais com os seus respectivos acompanhamentos e retornos e duas oficinas educativas. Houve prevalência do sexo feminino, de baixa escolaridade, baixa renda e casadas. As estratégias de educação em saúde e nutricional contínuas e participativas, com referência à realidade da população atendida é primordial para a mudança de comportamento alimentar e do estilo de vida. CONCLUSÃO: Constata-se, a necessidade de atividades educacionais contínuas com foco na promoção da saúde de idosos hipertensos, uma vez que estas, podem consequentemente manter niveis pressóricos adequados.
\end{abstract}

DESCRITORES: Hipertensão Arterial; Atenção Primária à Saúde; Educação em Saúde; Promoção da Saúde; Idosos.

\section{ABSTRACT}

OBJECTIVE: To describe the experience of educational health intervention for the control of arterial hypertension (SAH) in the elderly. METHODS: Descriptive study, type of experience report, of educational intervention actions developed with a group of hypertensive elderly people, between March and July 2019 in a UBS in the city of Paulistana - PI. RESULTS: 8 meetings were held: three lectures on healthy eating habits in the control of blood pressure levels; three nutritional consultations with their respective follow-ups and returns and two educational workshops. There was a prevalence of females, with low education, low income and married. Continuous and participatory health and nutritional education strategies, with reference to the reality of the population served, are essential for changing eating behavior and lifestyle. CONCLUSION: It appears that there is a need for continuous educational activities focused on promoting the health of hypertensive elderly people, as these, consequently, can maintain adequate blood pressure levels.

DESCRIPTORS: Arterial Hypertension; Primary Health Care; Health Education; Health Promotion; Elderly.

\section{RESUMEN}

OBJETIVO: Describir la experiencia de intervención educativa en salud para el control de la hipertensión arterial (HAS) en ancianos. MÉTODOS: Estudio descriptivo, tipo de relato de experiencia, de acciones de intervención educativa desarrolladas con un grupo de ancianos hipertensos, entre marzo y julio de 2019 en una UBS de la ciudad de Paulistana - PI. RESULTADOS: Se realizaron 8 reuniones: tres charlas sobre hábitos alimentarios saludables en el control de los niveles de presión arterial; tres consultas nutricionales con sus respectivos seguimientos y devoluciones y dos talleres educativos. Hubo un predominio de mujeres, con baja educación, bajos ingresos y casadas. Las estrategias continuas y participativas de educación en salud y nutrición, con referencia a la realidad de la población atendida, son fundamentales para cambiar la conducta alimentaria y el estilo de vida. CONCLUSIÓN: Parece que existe la necesidad de actividades educativas continuas enfocadas a promover la salud de los ancianos hipertensos, ya que estos pueden, consecuentemente, mantener niveles adecuados de presión arterial.

DESCRIPTORES: Hipertensión; Atención Primaria de Salud; Educación para la Salud; Promoción de la Salud; Ancianos.

RECEBIDO EM: 28/02/2021 APROVADO EM: 08/04/2021

\section{Jocenara Firme de Moura Santos Martins}

Nutricionista. Especialista em Nutrição Clínica e Saúde da Família e Comunidade pela Universidade Federal do Piauí/UFPI. Paulistana (PI), Brasil.

ORCID: 0000-0003-4867-0049 


\section{Jéssika Roberta Firme de Moura Santos}

Enfermeira. Mestranda do Programa de Pós-Graduação em Ciências e Saúde da Universidade Federal do Piauí/UFPI. Picos (PI), Brasil. ORCID: 0000-0003-1268-2400

\section{Lívia de Araújo Rocha}

Nutricionista. Mestre em Ciências e Saúde pela Universidade Federal do Piauí/UFPI. Picos (PI), Brasil. ORCID: 0000-0003-4595-410X

\section{Maurilo de Sousa Franco}

Enfermeiro pela Universidade Federal do Piauí/UFPI. Picos (PI), Brasil.

ORCID: 0000-0003-0808-3763

\section{Luana da Silva Amorim}

Acadêmica de Nutrição. Universidade Federal do Piauí/UFPI. Picos (PI), Brasil. ORCID: 0000-0001-7506-9334

\section{Luisa Helena de Oliveira Lima}

Doutora em Enfermagem. Professora Associada II da Universidade Federal do Piauí/CSHNB e do Programa de Pós-Graduação em Ciências e Saúde. Líder do Grupo de Pesquisa Inovação e Tecnologia no Ensino e no Cuidado em Saúde (ITECS)/ UFPI/CNPq. Picos (PI), Brasil.

ORCID: 0000-0002-1890-859X

\section{INTRODUÇÃO}

0 cenário brasileiro encontra-se com acentuado aumento de Doenças Crônicas Não Transmissíveis (DCNT) em decorrência da atual transição do perfil demográfico ${ }^{(1)}$. Nesse cenário, merece realce, a Hipertensão Arterial Sistêmica (HAS), caracterizada por condição clínica multifatorial de níveis elevados da pressão arterial (PA) podendo estar relacionada a alteraçôes na estrutura ou funcionalidade dos órgãos-alvo, além de alterações metabólicas, o que pode gerar elevado risco de eventos cardiovasculares fatais e não fatais ${ }^{(2)}$.

No contexto das DCNT, a HAS tem se destacado como importante problema de saúde pública apresentando prevalência nacional de 60\% dos casos na população idosa. Além disso, associado com o Diabetes Mellitus (DM), destacam-se como a principal causa de hospitalizações do Sistema Único de Saúde (SUS) devido a sua vulnerabilidade, o que ocasiona a necessidade de intervenções para promoção de saúde, qualidade de vida e prevenção desses agravos, cujo objetivo é tornar o processo de envelhecimento ativo, autossuficiente e saudável ${ }^{(1,3-5)}$.

A Organização Mundial da Saúde (OMS), ratifica a incidência de HAS sendo mais elevada em idosos, e destaca-se, os hábitos alimentares inadequados, como principal fator de risco para seu desenvolvimento. À vista disso, no acompanhamento do idoso com HAS, deve-se planejar e incrementar estratégias no âmbito do cuidado para o incentivo ao estilo de vida saudável, a execução de atividade física regular, e principalmente, adesão dos hábitos alimentares. Desse modo, uma forma de intervir para o controle da HAS, é o desenvolvimento de intervenções educativas ${ }^{(6)}$.

Assegura-se, na literatura científica o efeito positivo de intervenções multicompetentes como visita domiciliar, consulta individual e sessão educativa grupal incluindo desde modificações conscientes de hábitos alimentares, prática de atividade física e conhecimento do regime terapêutico são eficazes na redução de agravos de HAS, bem como a sua prevenção na população idosa $^{(7,8)}$.

É de grande relevância o aprimoramento da qualidade da assistência aos hipertensos, especialmente da atenção primária à saúde, com uma abordagem mais integral, com o objetivo de otimizar a adesão ao tratamento e realizar um acompanhamento mais criterioso ${ }^{(9)}$.

Dentro deste panorama, ações educativas são significativas para compor uma nova visão do processo saúde-doença, atentando para discussões a respeito das questốes que resultem em possíveis tomadas de decisões e estímulo no desenvolvimento de práticas que assegurem a adesão de um estilo de vida mais saudável da população idosa ${ }^{(3)}$.
Logo, o desenvolvimento de intervenções educativas como a descrita neste estudo, corroboram com as ações para controle da HAS a partir da promoção de hábitos saudáveis. Acredita-se, que intervenções educativas, tornam-se, estratégias de diálogo entre o profissional-serviço de saúde e paciente para a construção da autonomia e o gerenciamento da condição crônica, no caso desta experiência da HAS.

O presente estudo tem o objetivo de relatar a experiência de ações de educação em saúde em grupos de idosos hipertensos de uma Unidade Básica de Saúde da área urbana do município de Paulistana - PI, com a finalidade de controlar a hipertensão através do incentivo à adoção de hábitos alimentares saudáveis.

\section{MÉTODOS}

Trata-se de um estudo descritivo, do tipo relato de experiência, elaborado na conjuntura das ações de intervenção educativas e nutricionais desenvolvidas com um grupo de idosos hipertensos. As atividades foram desenvolvidas com aplicação prática em uma Unidade Básica de Saúde no município de Paulistana - PI nos meses de março a julho de 2019.

Para fins didáticos, o estudo foi implementado em etapas sequenciais: estudos teóricos do mecanismo da HAS e estratégias de intervenção; visita a UBS para formação de vínculo e inteirar-se a respeito da situação 
sociodemográfica, principais dificuldades em relação ao tratamento medicamentoso e aplicação prática de ferramentas de ações educacionais e nutricionais.

Inicialmente, realizou-se o cadastro dos idosos hipertensos, com a caracterização socioeconômica, dados pessoais, hábitos alimentares, medicação (identificação, dose e horário) em uso no tratamento da HAS para subsidiar na elaboração da in- tervenção. Desenvolveram-se as ações descritas no plano operacional (QUADRO 1 ), em encontros semanalmente em turno diurno matutino, totalizando 8 (oito) encontros em grupos com número médio de 10 a 13 participantes.

Por se tratar de um relato de experiência, não houve a utilização do Termo de Consentimento Livre e Esclarecido (TCLE).

\section{RESULTADOS}

Foram realizados 8 (oito) encontros com o grupo de idosos, distribuídos da seguinte forma: 3 (três) palestras dinâmicas e dialogadas sobre os beneficios dos hábitos alimentares saudáveis no controle dos níveis pressóricos; 3 (três) atendimentos nutricionais com o seu respectivo acompanhamento e retorno e 2 (duas) oficinas educativas "Sal de Ervas".

Quadro1- Plano Operacional das Ações Educativas e Nutricionais com o Grupo de Idosos da UBS. Paulistana (PI), 2019.

\begin{tabular}{|c|c|c|c|c|}
\hline $\begin{array}{c}\text { SITUAÇÃO } \\
\text { PROBLEMA }\end{array}$ & OBJETIVOS & METAS/ PRAZOS & AÇÕES/ ESTRATÉGIAS & RESPONSÁVEIS \\
\hline $\begin{array}{l}\text { Capacitação/ } \\
\text { Análise do } \\
\text { cenário }\end{array}$ & $\begin{array}{l}\text { - Capacitar os aca- } \\
\text { dêmicos voluntários } \\
\text { sobre a temática da } \\
\text { ação educativa (Grupo } \\
\text { de idoso hipertenso). } \\
\text { - Criar vínculo com a } \\
\text { comunidade. } \\
\text { - Reconhecer as prin- } \\
\text { cipais dificuldades na } \\
\text { adesão e manutenção } \\
\text { do tratamento de HAS. }\end{array}$ & $\begin{array}{c}\text { - Realizar no mês de } \\
\text { março/2019. }\end{array}$ & $\begin{array}{l}\text { Pesquisa em literatura científica sobre } \\
\text { a temática de HAS no público idoso. } \\
\text {-Elaboração do plano operacional da } \\
\text { intervenção educativa/nutricional. } \\
\text { - Elaboração de folder educativo } \\
\text { "HIPERTENSÃO". } \\
\text { - Preparaçãa de Oficina Educativa "Sal } \\
\text { de Ervas". }\end{array}$ & $\begin{array}{l}\text { - Nutricionista } \\
\text { - Enfermeira } \\
\text { - Acadêmicos de En- } \\
\text { fermagem e Nutrição } \\
\text { voluntários } \\
\text { (Supervisão da Nutricio- } \\
\text { nista/Enfermeira). }\end{array}$ \\
\hline $\begin{array}{c}\text { Hábitos } \\
\text { Alimentares } \\
\text { Inadequados } \\
\text { de idosos } \\
\text { hipertensos } \\
\text { da UBS do } \\
\text { município de } \\
\text { Paulistana } \\
\text { (PI). }\end{array}$ & $\begin{array}{l}\text { - Orientar o grupo de } \\
\text { idosos hipertensos } \\
\text { sobre a importância } \\
\text { da adoção de hábitos } \\
\text { alimentares saudáveis } \\
\text { como tratamento não } \\
\text { medicamentoso para } \\
\text { controle da HAS. } \\
\text { - Elaborar tecnologia } \\
\text { educativa em forma de } \\
\text { folder. }\end{array}$ & $\begin{array}{l}\text { Desenvolvimento } \\
\text { de três palestras } \\
\text { educativas e exposição } \\
\text { de um vídeo para } \\
\text { o grupo de idosos } \\
\text { hipertensos e seus } \\
\text { familiares durante o } \\
\text { mês de abril/2019 } \\
\text { abordando sobre o } \\
\text { benefício de uma } \\
\text { alimentação saudável } \\
\text { e escolha de alimentos } \\
\text { saudáveis. } \\
\text { - Elaboração de } \\
\text { tecnologia educativa } \\
\text { - folder intitulado } \\
\text { "Hipertensão" }\end{array}$ & $\begin{array}{l}\text { - Realização de palestras } \\
\text { educativas/ dinâmicas e exposição } \\
\text { de vídeos semanalmente, com } \\
\text { duração de } 60 \text { minutos, durante o } \\
\text { mês de abril/2019 na própria sede } \\
\text { da UBS, abordando sobre o benefício } \\
\text { de hábitos alimentares saudáveis. } \\
\text { Encontro 1: medidas associadas } \\
\text { ao preparo, bem como orientações } \\
\text { para promover uma alimentação } \\
\text { saudável para a pessoa idosa } \\
\text { Encontro 2: Medidas associadas ao } \\
\text { consumo de refeições diárias } \\
\text { Encontro3: Orientações acerca do } \\
\text { benefício da alimentação saudável } \\
\text { para o controle da pressão arterial, } \\
\text { proporcionando um estilo de vida } \\
\text { saudável e uma melhor qualidade de } \\
\text { vida ao grupo de idosos hipertensos. } \\
\text { *Uso de recursos audiovisuais } \\
\text { - Distribuição durante o } 3^{\circ} \\
\text { encontro na UBS folder educativo } \\
\text { e ilustrativos aos idosos sobre } \\
\text { os alimentos aceitos no controle } \\
\text { da HAS, bem como orientações } \\
\text { adicionais (o conceito de HAS, } \\
\text { sintomas, fatores de risco e } \\
\text { alimentos a serem evitados e } \\
\text { aceitos na alimentação). }\end{array}$ & $\begin{array}{l}\text { - Nutricionista } \\
\text { - Enfermeira } \\
\text { - Acadêmicos de } \\
\text { Nutrição e Enfermagem } \\
\text { voluntários (Supervisão da } \\
\text { Nutricionista/Enfermeira). } \\
\text { - Nutricionista } \\
\text { - Enfermeira } \\
\text { - Acadêmicos de } \\
\text { Nutrição e Enfermagem } \\
\text { voluntários (Supervisão da } \\
\text { Nutricionista/Enfermeira). }\end{array}$ \\
\hline
\end{tabular}




\begin{tabular}{|c|c|c|c|}
\hline $\begin{array}{l}\text { - Realizar aconse- } \\
\text { Ihamento nutricional } \\
\text { adequado aos idosos. }\end{array}$ & $\begin{array}{l}\text { - Realizar atendimento } \\
\text { nutricional individual } \\
\text { nos meses de maio a } \\
\text { julho/2019 aos idosos } \\
\text { hipertensos da UBS. } \\
\text { - Realizar consulta } \\
\text { nutricional de retorno aos } \\
\text { idosos hipertensos. }\end{array}$ & $\begin{array}{l}\text { - Realização de atendimento nutricional nos me- } \\
\text { ses de maio a julho/2019 e o retorno dos idosos } \\
\text { hipertensos na sede do NASF I. } \\
\text { - Anamnese alimentar; } \\
\text { - Avaliação Antropométrica; } \\
\text { - Prescrição e orientação de plano alimentar cen- } \\
\text { trada nas mudanças de impacto na diminuição da } \\
\text { PA, perda de peso, aumento de consumo de frutas } \\
\text { e vegetais e redução do consumo de sódio. } \\
\text { - Acompanhamento das mudanças dietéticas e } \\
\text { evolução antropométrica. }\end{array}$ & $\begin{array}{l}\text { - Nutricionista. } \\
\text { - Acadêmico de Nutri- } \\
\text { ção (Supervisionado } \\
\text { pela Nutricionista). } \\
\text { - Nutricionista. } \\
\text { - Acadêmico de Nutri- } \\
\text { ção (Supervisionado } \\
\text { pela Nutricionista). }\end{array}$ \\
\hline $\begin{array}{l}\text { - Estimular o uso do } \\
\text { sal de ervas como } \\
\text { opção de tempero no } \\
\text { auxílio do controle da } \\
\text { HAS. }\end{array}$ & $\begin{array}{l}\text {-Desenvolver duas Ofi- } \\
\text { cinas Educativas "Sal de } \\
\text { Ervas" para os idosos hi- } \\
\text { pertensos durante o mês } \\
\text { de junho e julho/2019. }\end{array}$ & $\begin{array}{l}\text { - Realizar durante o mês de junho e julho/2019 na } \\
\text { sede da UBS Lagoa, duas oficinas "Sal de Ervas", } \\
\text { para a preparação e distribuição de amostras } \\
\text { do sal aos hipertensos em substituiçãa ao sal } \\
\text { convencional. } \\
\text { - Aceitabilidade do sal de ervas pelo grupo de } \\
\text { idoso hipertensos como tempero na alimentaçãa. }\end{array}$ & $\begin{array}{l}\text { - Nutricionista } \\
\text { - Enfermeira } \\
\text { - Acadêmicos de Nu- } \\
\text { trição e Enfermagem } \\
\quad \text { voluntários. }\end{array}$ \\
\hline $\begin{array}{l}\text { - Monitoramento da } \\
\text { Pressão Arterial (PA). }\end{array}$ & $\begin{array}{c}\text { - Aferir a Pressão Arterial } \\
\text { (PA) do idoso cadastrado } \\
\text { no grupo de hipertensos } \\
\text { participantes das ações } \\
\text { educativas, nos meses de } \\
\text { março a julho/2019. }\end{array}$ & $\begin{array}{l}\text { Aferição da PA dos idosos pertencentes ao grupo } \\
\text { de idosos durante as intervenções educativas } \\
\text { (palestras educativas e dinâmicas; distribuição e } \\
\text { aconselhamento do folder educativo "Hiperten- } \\
\text { são", Atendimento e Retorno Nutricional e Oficina } \\
\text { "Sal de Ervas"). }\end{array}$ & $\begin{array}{l}\text { - Enfermeira } \\
\text { - Acadêmico de Enfer- } \\
\text { magem (com supervi- } \\
\text { são da Enfermeira) }\end{array}$ \\
\hline
\end{tabular}

Inicialmente foi feito o reconhecimento das principais dificuldades na adesão, manutenção e consequentemente os fatores que interferiam no desfecho satisfatório do tratamento da HAS no público idoso, chegando a conclusão da importância de concentrar em ações educativas e nutricionais e subsidiaram a construção da tecnologia (folder) a partir dos pontos principais obtidos no reconhecimento e primeiros encontros com o grupo de idosos.

O primeiro encontro teve como objetivo abordar a temática "Alimentação saudável na Terceira Idade", embasada pelo Manual Alimentação Saudável para a Pessoa Idosa do Ministério da Saúde, abordando medidas associadas ao preparo de refeições diárias, bem como orientaçóes para promover uma alimentação saudável para a pessoa idosa. Utilizou-se a dinâmica de sessão educativa presencial, contando com o uso de meios audiovisuais (ilustrações, vídeos, figuras) proporcionando um melhor entendimento para o público idoso sobre os seguimentos assim descritos: cuidados na compra e armazenamento dos alimentos, cuidados com a higiene e manuseio dos alimentos e cuidados da preparação dos alimentos.
Segundo encontro tratou-se "Medidas Associadas ao Consumo dos Alimentos", abordando as seguintes questões: escolha do alimento saudável, desestímulo do uso de sal à mesa, ingestão adequada de líquidos. O Terceiro encontro finalizou com as "Orientações Nutricionais centradas na diminuição da Pressão Arterial”, utilizou-se como forma de auxiliar a intervenção uma tecnologia educativa desenvolvida pela equipe de profissionais Nutricionista e Enfermeiros folder "Hipertensão" com linguagem de fácil compreensão, construído com as seguintes secções a saber: abordagem direta de definição hipertensão, sinais e sintomas, fatores de risco, dicas de hábitos saudáveis como o que preferir e evitar na alimentação a fim de proporcionar uma melhor qualidade de vida e um estilo de vida saudável aos idosos hipertensos, todo o conteúdo estabelecido pela literatura científica.

$\mathrm{Na}$ etapa seguinte da intervenção, realizou uma oficina "Sal de Ervas", a mesma foi distribuída em dois encontros com o intuito de promover a interação entre os idosos e profissionais de saúde.

\section{DISCUSSÃO}

As estratégias de educação em saúde e nutricional contínuas e participativas, com referência à realidade da população atendida, são primordiais para a mudança de comportamento alimentar e do estilo de vida a curto, médio e longo prazo como meio de superar a baixa adesão ao tratamento não farmacológico da $\mathrm{HAS}^{(10)}$.

A participação na intervenção foi similar aos encontrados em outros estudos, que apontam o maior público de ações educativas sendo composta pelo sexo feminino, de baixa escolaridade e baixa renda e casada ${ }^{(11-13)}$.

No que se refere ao gênero, as mulheres por sua vez, apresentam uma maior predisposição ao autocuidado, busca pelo serviço de saúde e percepção de doenças, tendo assim níveis de incidência elevado de HAS em relação ao público masculino ${ }^{(14)}$.

Em relação ao primeiro encontro, foi abordado o tema "Alimentação saudável na Terceira Idade", os aspectos relacionados à nutrição do idoso são resultados refletidos a partir do processo fisiológico como as alterações anatômicas e funcionais do próprio en- 
velhecimento. Dado isso, a importância de uma alimentação balanceada, rica e variada na busca de contribuir para uma maior longevidade com qualidade de vida ${ }^{(15)}$.

Diante disso, entre as abordagens de tratamento da hipertensão arterial, mudanças no estilo de vida, constituídas por modificações na dieta e aumento da atividade física, são eficazes na redução da pressão arterial, na prevenção da hipertensão e suas sequelas ${ }^{(8)}$. Isso demonstra a necessidade de trabalhar fundamentalmente o desenvolvimento contínuo de ações direcionadas aos determinantes sociais em saúde principalmente nas populações mais vulneráveis ${ }^{(16)}$.

Atenta-se para as variadas modalidades de tecnologias educacionais listadas na literatura, sendo elas expositivas, dialogadas, audiovisuais, táteis e impressas como o Folder Educativo "Hipertensão", distribuído ao final do terceiro encontro, têm se indicado como um significativo promotor de saúde e eficaz na promoção de hábitos alimentares saudáveis na HAS como também valoroso instrumento de interação profissional e população ${ }^{(4)}$.

O tratamento farmacológico de maneira isolada não controla as cifras de tensão arterial, devido aos maus hábitos de vida e alimentação, pois a exposição diária a alguns fatores, como consumo excessivo de sódio, baixa ingestão de potássio, sobrepeso, obesidade, sedentarismo e a ingestão de álcool aumentam a pressão arterial gradualmente ${ }^{(17)}$. Ainda assim, Malachias et al.,(18), traz que as pessoas, geralmente, não associam esses aspectos como fator de risco para essa doença, contribuindo assim para agrande prevalência e descontrole.

\section{CONCLUSÃO}

Por se tratar de um grupo mais vulnerável e muitas vezes com diversas limitações, as intervenções educativas são fundamentais para a mudança de hábitos não só alimentares, mas também a mudança de adoção de atitudes de risco, influenciando assim, de forma direta a saúde dessa população, especialmente para o controle da pressão arterial. Contudo, evidencia-se a importância da realização de atividades como estas para a promoção da saúde na terceira idade.

\section{REFERÊNCIAS}

1. Soares EMF, Moraes GLA, Feitosa JÁ, Pessoa PMD, Oliveira VS. Influência do bem-estar psicossocial do idoso no enfrentamento de adversidades em saúde: relato de experiência. Saúde Coletiva. 2020; 10(59): 4162-4166.

2. Cardoso FN, Domingues TAM, Silva SS, Lopes JL. Fatores de risco cardiovascular modificáveis em pacientes com hipertensão arterial sistêmica. REME - Rev Min Enferm. 2020; 24:e-1275. DOI: 10.5935/14152762.20200004

3. Azevedo PRA, Sousa MM, Sousa NF, et al. Ações de educação em saúde no contexto das doenças crônicas: revisão integrativa. Rev Fund Care Online. 2018; 10(1):260-267. DOI: http://dx.doi.org/ 10.9789/2175-5361.2018.v10i1.260-267

4. Santos LF, Silva RC, Santos NSS, Mutti CF, Oliveira LMAC. Participation in a group and quality of life in hypertensive perspectives. J Nurs UFPE On Line [Internet]. 2016; 10(8):2886-94. DOl: http:/dx.doi. org/10.5205/1981-8963-v10i8a11357p2886-2894-2016

5. Silva KM, Santos SMA, Souza AlJ. Reflexões sobre a Necessidade do Cuidado Humanizado ao Idoso e familia. Sau. \& Transf. Soc. 2014;5(3):20-4.

6. Afonso VLM, Garcia RR Sinato, CM, Nascimento RG, Carmo FS. Educação em saúde e estratégias utilizadas para prevenção e controle da hipertensão arterial com idosos. Revista Baiana de Saúde Pública. 2018; 42(2): 368-381 DOl: 10.22278/2318-2660.2018.v42.n2.a2458

7. Jafar TH, Gandhi M, de Silva HA, Jehan I, Naheed A et al. A Community-Based Intervention for Managing Hypertension in Rural South Asia. New England Journal of Medicine. 2020; 382(8), 717-726. DOI https:/ doi.org/10.1056/NEJMoa1911965

8. OparilS, Acelajado MC, Bakris GL, BerlowitzDR, CífkováR, Dominiczak AF, Grassi G, Jordan J, Poulter NR, Rodgers A, Whelton PK. Hypertension. Nat Rev Dis Primers. 2018 Mar 22;4:18014. DOI: 10.1038/ nrdp.2018.14. PMID: 29565029; PMCID: PMC6477925.

9. Nobre ALCSD, Lima CDA, Oliveira MJLD, Vieira DDMA, Martelli Júnior $\mathrm{H}$, Costas SDM. Hipertensos assistidos em serviço de atenção secundária: risco cardiovascular e determinantes sociais de saúde. Cad- ernos Saúde Coletiva, v. 28, n. 3, p. 334-344, 2020.

10. Machado Juliana Costa, Cotta Rosângela Minardi Mitre, Moreira Tiago Ricardo, Silva Luciana Saraiva da. Análise de três estratégias de educação em saúde para portadores de hipertensão arterial. Ciênc. saúde coletiva [Internet]. 2016; 21( 2 ): 611-620. DOl:. http://dx.doi. org/10.1590/1413-81232015212.20112014.

11. Ferreira DN, Matos DL, Loyola Al Filho. Ausência de consulta médica de rotina entre idosos hipertensos e/ou diabéticos: um estudo epidemiológico baseado na Pesquisa Nacional por Amostra de Domicilios 2008. Rev Bras Epidemiol. 2015;18(3):578-94.

12. Mendes TAB, Goldbaum M, Segri JN, Barros MBA, César CLG, Carandina $\mathrm{L}$. Factors associated with the prevalence of hypertension and control practices among elderly residents of São Paulo city, Brazil. Cad Saúde Pública. 2013;29(11):2275-86.

13. Dias EG, Silva EJF, Lima FN, Anjos ECF, Alves JCS. Caracterização dos hipertensos e fatores dificultadores na adesão do idoso ao ratamento medicamentoso da hipertensão. Rev Interdisciplin. 2015;8(3):39 49.

14. Almeida AV, Mafra SCT, Silva EP, Kanso S. A feminização da velhice: em foco as características socioeconômicas, pessoais e familiares das idosas e o risco social. Textos Contextos. 2015;14(1):115-31.

15. Moreira RP, Ferreira GO, Felício JF, Lima PA de, Gomes TF, Oliveira FBB de. Educação em saúde no domicilio de idosos hipertensos e diabéticos. Rev enferm UFPE on line. 2020;14: e 245034 DOI: https://doi. org/10.5205/1981-8963.2020.245034.

16. Andrade SSDA, Stopa SR, Brito AS, Chueri PS, Szwarcwald CL, Malta DC. Self reported hypertension prevalence in the Brazilian population: analysis of the National Health Survey, 2013. Epidemiol Serv Saude, v. 24, n. 2 p. 297-304, 2015.

17. Poulter NR, Prabhakaran D, Caulfield M. Hypertension. Lancet, v. 386, 801-812 2015.

18. Malachias MVB et al. 7a Diretriz brasileira de hipertensão arterial. Arquivos Brasileiros de Cardiologia, Rio de Janeiro, v. 107, n. 3, supl. 3, set. 2016. 